\title{
Simple Approaches to Reduce Radiation in the Electrophysiology Laboratory
}

\author{
Serkan Cay ${ }^{1}$, Ozcan Ozeke ${ }^{1}$, Firat Ozcan ${ }^{1}$, Serkan Topaloglu ${ }^{1}$, and Dursun Aras ${ }^{1}$ \\ ${ }^{1}$ University of Health Sciences, Ankara City Hospital
}

April 28, 2020

To the Editor,

We have read with great interest the article entitled 'Electrogram-only guided approach to His bundle pacing with minimal fluoroscopy: A single-center experience' by Zanon et $\mathrm{al}^{1}$ in the latest issue of the journal. We would like to thank the authors for their study regarding the use of electrograms during implantation of the His lead with minimal or no fluoroscopy. Related radiation dose parameters such as fluoroscopy time and dose area product (DAP) were also presented. Radiation dose related to the fluoroscopy system is generally dependent on various autoregulated variables including the tube voltage, the tube current and the pulse duration. In addition, various programmable settings including the frame rate, collimation, and magnification are used to regulate the amount of radiation although the number of variables which can be programmed in the manufacturer specific system varies. Fluoroscopy time should not be an estimation factor for radiation dose because its correlation with other standard radiation measures is weak. Manufacturer specific calculations, either total time while pressing on the fluoroscopy pedal or only a sum of fluoroscopic pulses, make the radiation dose difficult to estimate. The air kerma area product, or DAP $\left(\mathrm{Gy} . \mathrm{cm}^{2}\right)$ is accepted as the most accurate measurement of radiation energy given for fluoroscopic purposes. Roughly, the effective dose (ED) (mSv) of fluoroscopic source of radiation can be calculated using a conversion factor of 0.20 from the DAP measurement. For instance, routine single/dual-chamber pacemaker/defibrillator implantation has an average ED of $4 \mathrm{mSv}$, compared to an average ED of nearly $20 \mathrm{mSv}$ for a cardiac resynchronization therapy device implantation. ${ }^{2}$ The frame rate, collimation and magnification should be properly set to reduce the DAP/ED values.

The frame rate seems as the most important programmable factor related to the radiation dose. Continuous fluoroscopy has generally the frame rate of [?]30 frames per second (fps), which is associated with increased radiation exposure. Therefore, the frame rate should be set as low as achievable. Decreasing the frame rate from 30 fps to 7.5 fps reduces the ED by $75 \%$. Compensatory tube current increase generally occurs during pulsed fluoroscopy, which is associated with the same number of x-rays produced during continuous fluoroscopy. However, significantly decreased number of pulses cause significant reduction of radiation exposure in a linear way. The frame rates as low as [?]3 fps should be used during diagnostic and ablation procedures and device implantations in the electrophysiology laboratory although reduced temporal resolution. ${ }^{2,3}$ Furthermore, configuring the frame rate [?]1 fps using the ECG triggering or pacing artifact can be the best approach. Configuration of the pulsed fluoroscopy at the lowest frame rate needed has the greatest potential for ensuring radiation dose at the lowest level.

Properly configured and applied collimation, reduction of radiation to an anatomical area that requires imaging, has an important role in reducing both the direct and the scattered radiation. Collimation, especially the asymmetric one, reduces visualization area and decreases the DAP/ED in a linear way. During the procedure, the use of collimation restricts the view on the cardiac contour. After viewing the whole anatomy, 
re-collimation should be performed.

Another option for reducing the radiation dose is to set the largest field of view, or the smallest magnification, possible. In the smallest field of view mode, or the largest magnification mode, a smaller radiation area is projected in the image intensifier and, a larger image is produced on the screen. This process is related to a lower radiation output and a dimmer image. The fluoroscopy unit has an automatic system for controlling the brightness sensed. Therefore, the automatic system compensates for the lower brightness by increasing radiation exposure from the tube. With the use of magnification, the radiation dose increase in flat-panel detectors is lower compared to the image-intensifiers, however it is still remarkable. ${ }^{4}$

In the light of all above-mentioned settings, the fluoroscopy time should not be used as an important variable measuring the radiation dose. Instead, the DAP/ED values should be reported.

Keywords: dose area product/effective dose; fluoroscopy; programmable; radiation dose

\section{Funding}

None.

\section{Conflict of interests}

The authors declare that there are no conflict of interests.

\section{References}

1 - Zanon F, Marcantoni L, Zuin M, et al. Electrogram-only guided approach to His bundle pacing with minimal fluoroscopy: A single-center experience. J Cardiovasc Electrophysiol . 2020;31(4):805-812.

2 - Heidbuchel H, Wittkampf FH, Vano E, et al. Practical ways to reduce radiation dose for patients and staff during device implantations and electrophysiological procedures. Europace . 2014;16(7):946-964.

3 - Thibault B, Macle L, Mondésert B, et al. Reducing radiation exposure during procedures performed in the electrophysiology laboratory. J Cardiovasc Electrophysiol . 2018;29(2):308-315.

4 - Nachiappan AC, Horn GL, Spann SC, et al. Operator radiation dose reduction during fluoroscopic interventional procedures. J Am Coll Radiol . 2015;12(5):527-530. 